\title{
Analysis of Lung and Heart Sound Using Smartphone Stethoscope
}

\author{
Eui-bung Jeoung ${ }^{1}$, Soo-mi Hong ${ }^{2}$, Hyung-ki Choi ${ }^{3}$, and Kee-young Park ${ }^{4}$ \\ ${ }^{1}$ Dept. Automotive \& Mechanical Engineering, Howon Univ., Kunsan, Korea \\ ${ }^{2}$ Corresponding author, Associate Professor, Major in Real Estate Studies, Faculty of \\ Regional Development, Kongju Nat'l University. Yesan, Chungnam, Korea \\ ${ }^{3,4}$ Dept. R\&D, Sunmeditec co., ltd., Jeonju, Korea \\ ${ }^{1}$ bung4524@hanmail.net, ${ }^{2}$ nayasoo3@hotmail.com, ${ }^{3}$ hkchoi@sunmeditec.com, \\ ${ }^{4}$ kypark@sunmeditec.com
}

\begin{abstract}
Korea and the world population are suffering from heart and lung disease due to aging. There are only few objective data regarding to bronchial or lung sounds. Therefore, it is necessary to normalize the data for breathing sounds objectively. In this paper, we analyze lung data using algorithm PCA (Principal Component Analysis) and AVRLCR (Average Level Crossing Rate) and then present an objective data about asthma and pneumonia. Peak frequency and AVRLCR value of spectrum show the significant differences depend on the type of diseases. And based on the period of the waveform, the waveform is displayed by autocorrelation and the pulse rate is displayed in real-time.
\end{abstract}

Keywords: Heart and lung disease, PCA (Principal Component Analysis), AVRLCR (Average Level Crossing Rate), Autocorrelation, Pulse rate

\section{Introduction}

There are many subjective opinions of doctors in diagnosing heart and pulmonary disease with a stethoscope. However, the smart stethoscope proposed here displays heart and pulmonary disease as waveforms and spectrograms to provide objective diagnosis.

Using auscultation in pulmonary medicine has not theoretically been developed yet, so it calls for the quantitative analysis of the breath sound [1][2]. At the same time, lung sound has sonant to differ according to circumstance in despite of same patients. The purpose of this study was to categorize normal person and patients who has pneumonia. The diagnosis was made using the smart stethoscope and characteristics of diseases was confirmed by PCA and AVRLCR algorithm.

\section{Principal component analysis \& AVRLCR}

\subsection{Analysis and algorithm}

PCA is a versatile technique and has been used widely in signal processing for various applications such as dimensionality reduction, data compression, and feature extraction. This

Article history:

Received (June 1, 2019), Review Result (August 15, 2019), Accepted (September 26, 2019) 
is especially important in lung sounds processing, because it can be used to decorrelate spectral correlation. And PCA is the optimal linear method as a figure of merit.

Kernel PCA have been proposed by Schölkopf [3][4][5].

We, here, give an outline of kernel PCA.

Given a set of $\mathrm{M}$ centered observation, $x_{i} \in R(i=1,2, \ldots, \mathrm{M})$. We consider a nonlinear mapping, $\Phi$, to feature space $F$.

$$
\Phi: R \rightarrow F, x \rightarrow \Phi(x)
$$

In order to compute dot products of the form $\left(\Phi() \cdot \Phi\left(x_{i}\right)\right)$, we define kernel function $K$,

$$
K\left(x_{i}, x_{j}\right)=\Phi\left(x_{i}\right) \cdot \Phi\left(x_{j}\right)
$$

which allow us to compute the value of the dot product in F without having to carry out the map $\Phi$.

Defining an $M x M$ matrix $K$ by $K_{i j}=K\left(x_{i}, x_{j}\right)$, we need to diagonalize $\mathrm{K}$ to obtain eigenvalue, $\lambda$, and eigenvector, $\alpha$, in $F$.

$$
M \lambda \alpha=K \alpha
$$

$\lambda_{1} \leq \lambda_{2} \leq \cdots \leq \lambda_{M}$ denote eigenvalues, and $\alpha^{n}$ the corresponding complete set of eigenvectors which have elements of $\alpha_{1}^{n}, \alpha_{1}^{n}, \cdots, \alpha_{M 1}^{n}$. We normalize $\alpha^{n}$ as

$$
I=\lambda_{k}\left(\alpha^{n} \cdot \alpha^{n}\right) \text { for all } k=1, \ldots, M
$$

Let $x$ represent a test point, $\mathrm{n}$-th kernel principal components are

$$
\alpha_{i}^{n} \cdot K\left(x_{i}, x_{j}\right)
$$

Kernel function is often used as follows:

1. Polynomial kernel

$$
K\left(x_{i}, x_{j}\right)=\left(x_{i} \cdot x_{i}+r\right)^{d}
$$

2. Gaussian kernel

$$
K\left(x_{i}, x_{j}\right)=\exp \left[-c\left|x_{i}-x_{j}\right|^{2}\right]
$$

Here, $d, r$, and $c$ are parameters [6].

Next, this section is devoted to a description of the LCR and AVRLCR. In general, one pitch period of a thoracic waveform is used as the unit in speech synthesis by editing speech elements. The reason the level cross processing is explained here is because it is the simplest case. In this system, one pitch period of lung sounds waveform is stored as a signal element in the form of level cross interval sequence. Therefore, level crossing rate is as follows.

$$
L_{n}=\sum_{m=0}^{N-1}\left|\operatorname{sgn}\left[x(n-m)-L_{t h}\right]-\operatorname{sgn}\left[x(n-m-1)-L_{t h}\right]\right|
$$

And average level crossing rate is

$$
\text { AVRLCR }=\sum_{m=0}^{N-1}[L(n) / \text { length }]
$$

It needs elements to represent the transitional parts for length. These transition elements are produced by various combinations of the first and second formants. Each of these elements is represented by LCR, on the average, and all intervals in the level cross are quantized by 12 bits.

\subsection{Test methods}


A block diagram of the pneumonia data extraction system is shown in [Figure 1]. The sounds of the chest are obtained by air-coupled microphones (Header sensor). The signal input to the sensor is sent to the preamplifier and the high gain amplifier, and is then sent to a low pass filter (third order $1000 \mathrm{~Hz}$ ) and an envelope extractor circuit to extract the characteristics of the pneumonia. The envelope extractor consists of an absolute value amplifier, a peak detector circuit and an S/H amplifier. The peak detector acquires the maximum amplitude of the pneumonia absolute value during the sampling period. The peak value is sent in $\mathrm{S} / \mathrm{H}$ and the peak detector is reset. A sequence generates the necessary control signals from the digital control clock.

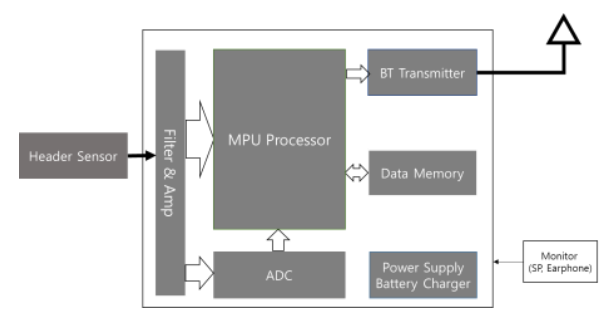

Figure 1. Block-diagram of pneumonia data extraction system

The system is based on a smartphone with a data acquisition boards (SMT_5000). The board has one channel analog inputs which are sequentially digitized with 12 bit resolution during the sampling period. The board also has digital I/O ports which are used to control the clock frequency and the gain of the amplifiers. The digitized signals are transferred by I2C ports to the memory and finally stored on the hard disk [7][8][9][10][11].

The following procedure was used to calculate the level crossing rate energy. First, the header sensor was calibrated independently from the system by using an audio analyzer. The mean sensitivity of a microphone (header sensor) was $12 \pm 3 \mathrm{mV} / \mathrm{Pa}$. Their frequency bandwidth was flat from 20 to $10 \mathrm{KHz}$ by cut off frequency $\pm 3 \mathrm{~dB}$. Preamplifiers of header sensor microphone are calibrated by applying a $1 \mathrm{mV}$ sinusoidal signal to the input and storing the A/D output value corresponding to each gain level. The transfer function is approximated by a linear feedback of these values. The feedback constants and the microphone sensitivity of each input are stored in a memory. During the data acquisition, each lung sounds data is calculated with an average level crossing rate computed from the constants of its inputs.

\subsection{Results and discussion}

10 recordings were performed by each disease with an acquisition time of 1 minute on 5 positions (left, right, center of lung and left and right below the back) and a sampling rate of $2 \mathrm{KHz}$ per input at Jesus Hospital in Jeonju city. In this paper, the number of numerical data samples of a lung sounds are 34000 and training set per disease is 10. And block diagram of PCA algorithm is shown in [Figure 2]. 


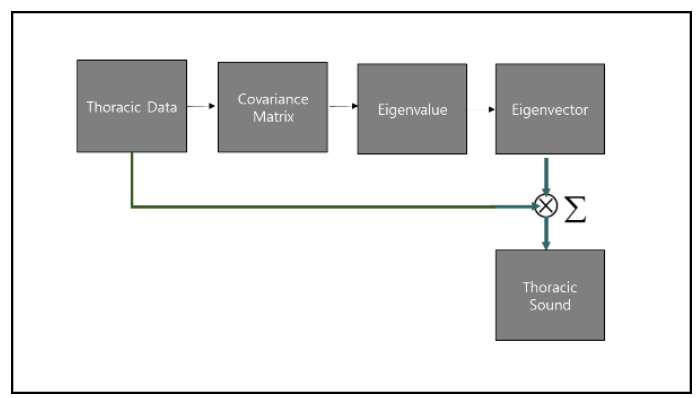

Figure 2. Flowchart of PCA algorithm

[Figure 3] shows normalization data of 10 thoracic sound sets by each asthma patient. Row axis is sampling number and column axis is the amplitude of sampling. At the same time, [Figure 4] shows original data of 5 thoracic sound sets by each asthma patient.

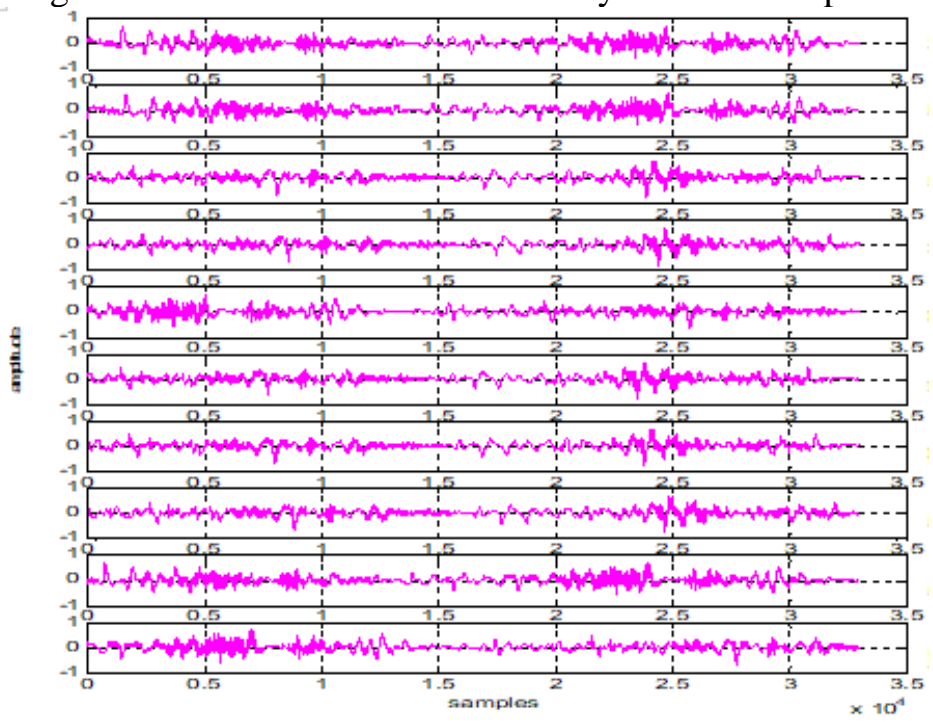

Figure 3. Thoracic sound sets of asthma patient

The first picture includes the most common element of each thoracic trouble data in [Figure 4]. And the 10th data preserved the smallest common element in [Figure 4]. For the same principle, the first picture includes the most common element of each disease.

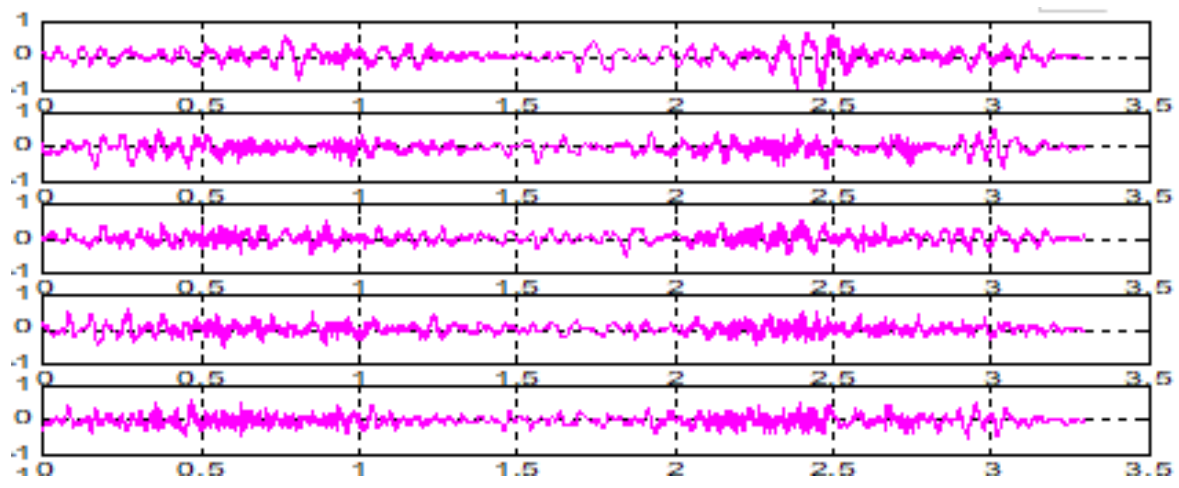

Figure 4. Original thoracic sound of asthma patient 
To obtain first vector value of PCA, we applied a simulation through software program. As a result, the original thoracic sound vector values are shown in [Table 1].

Table 1. Implemented code of blood pressure

\begin{tabular}{|c|c|c|c|}
\hline & Asthma & Pneumonia & Bronchitis \\
\hline \multirow{4}{*}{} & -0.0787 & -0.0654 & 0.0937 \\
\cline { 2 - 4 } & -0.0355 & 0.1639 & 0.0570 \\
\cline { 2 - 4 } & 0.5239 & 0.1310 & 0.0298 \\
\cline { 2 - 4 } & -0.3830 & -0.5752 & 0.0240 \\
\cline { 2 - 4 } $\begin{array}{c}\text { Thoracic } \\
\text { Sound } \\
\text { Space } \\
\text { Vector } \\
\text { Values }\end{array}$ & 0.0023 & -0.0257 & -0.4490 \\
\cline { 2 - 4 } & -0.4130 & -0.2570 & -0.1525 \\
\cline { 2 - 4 } & 0.4845 & -0.0469 & -0.1973 \\
\cline { 2 - 4 } & 0.3854 & -0.5677 & -0.5966 \\
\cline { 2 - 4 } & -0.0582 & -0.5431 & -0.6011 \\
\hline
\end{tabular}

[Figure 5] shows comparison of LCR and AVRLCR for each disease. Furthermore, [Figure 6] shows obvious difference in AVRLCR for detecting each disease.

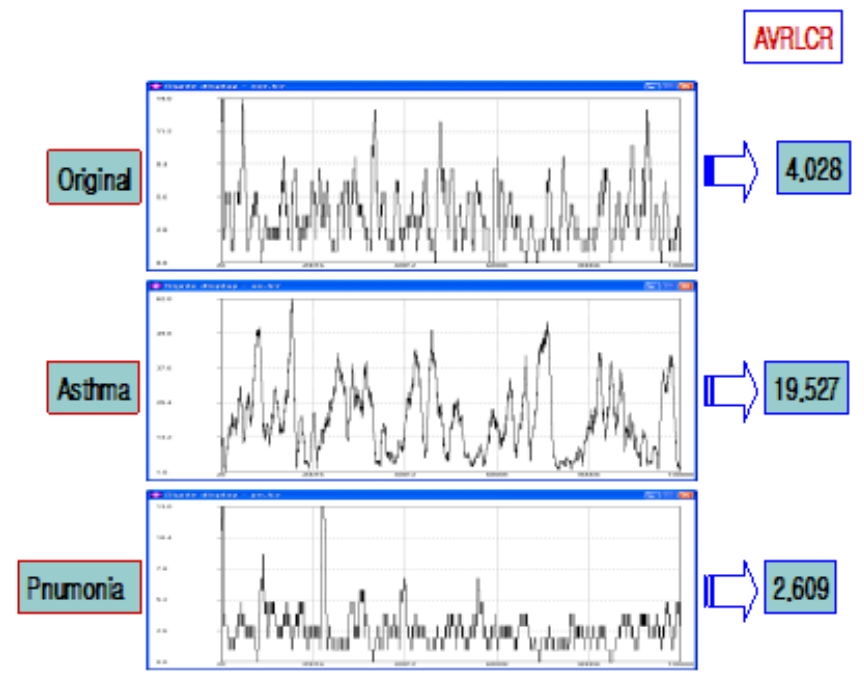

Figure 5. Comparison of LCR and AVRLCR for each disease 


\section{Level 0 AVRLCR}

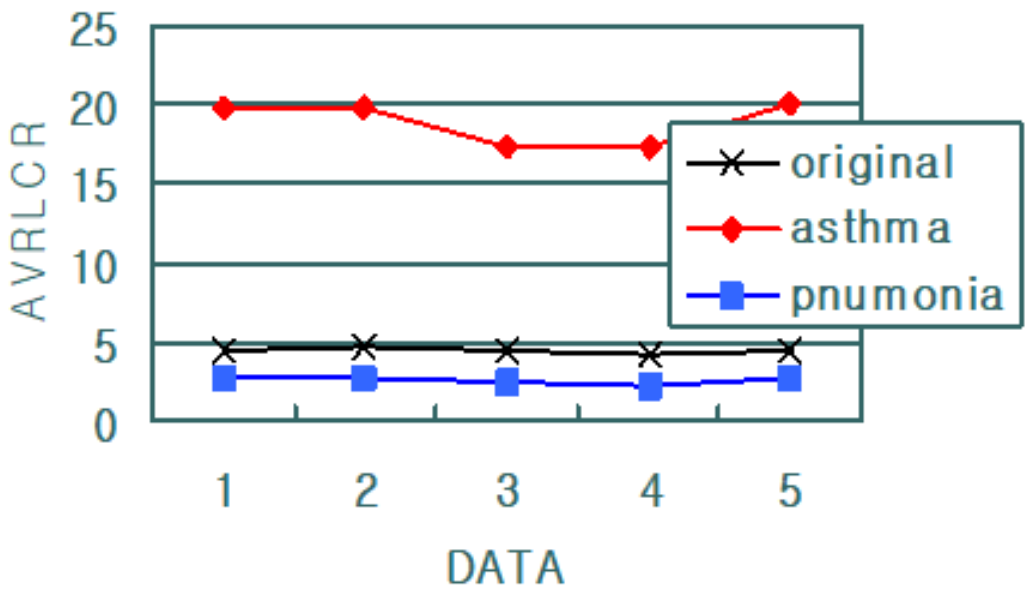

Figure 6. Comparison of AVRLCR for each disease

\subsection{Display and pulse rate}

The autocorrelation function (10) was used to calculate and display the waveforms and pulse rates of the stethoscope sounds (such as heart sounds and thoracic sounds). And a firmware program was applied to accurately display the waveform on the time domain. The autocorrelation function (1) was used to calculate the absolute value which is the positive value. The program accurately displays the period of the waveform in the time domain and calculates the heart rate by calculating the period. [Figure 7] shows the pulse rate (Heart rate) and waveform in real-time.

$$
R_{f f}(\tau) \triangleq \int_{t_{0}}^{t_{0}+T} f(t+\tau) \overline{f(t)} d t
$$

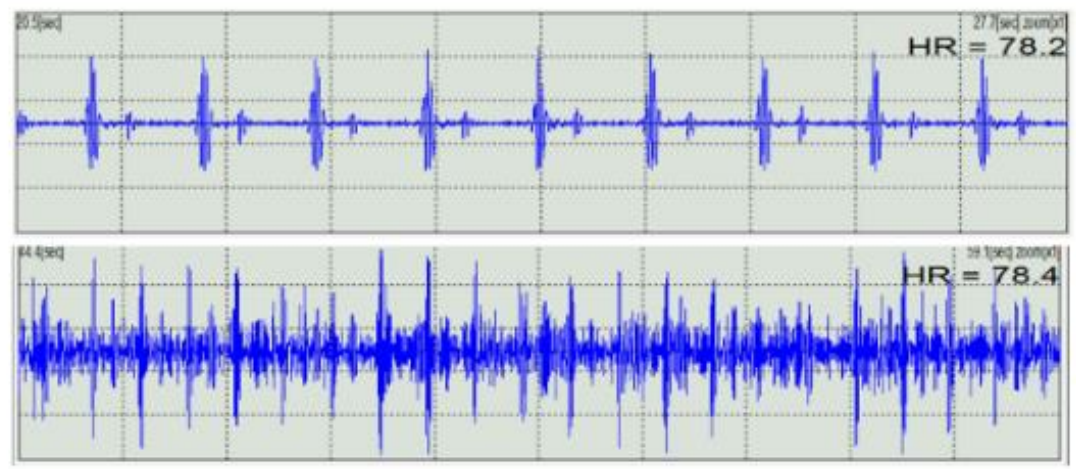

Figure 7. Heart rate (HR) and waveform

\section{Conclusion}

In the experiment for lung sounds, the structure of hardware system and design through programming are introduced. In this paper, we propose characteristic lung sounds using PCA and AVRLCR for objective data of lung sounds. 
[Figure 5] and [Figure 6] are shown various data for each disease which are detected as the useful lung sound data in diagnosis.

Respiratory physicians evaluated the detected lung sound data and we have attained results of a feature that can determine the disease.

Considering the above results, it is necessary to study for more accurate hardware structure and software lung sound of the system.

In addition, more reference DB for solving the reliable lung sound algorithm should be designed as complete hardware system. We are trying to analyze the constraints and various distortions of the hardware for more useful design.

Also, a smartphone u-healthcare diagnosis system using VICM is constructed and we have developed a convenient smartphone diagnostic system that can diagnose a person's health condition anytime and anywhere without being restricted by place and time.

\section{Acknowledgement}

This research was supported by Howon University of academic research funding.

\section{References}

[1] J.S. Choi, "Respirstory sound," pp.67-70, (1994)

[2] R. Loudon and PLH. Murphy, "Lung sounds,” Am Rev Respir Dis, pp.663-673, (1984)

[3] A. Papoulis. Probability, "Random variables, and stochastic processing," McGraw Hill, 3rd edition, (1991)

[4] B. Schölkopf, A. Smola, and K.R. Möller, "Nonlinear components analysis as a kernel eigenvalue problem," Technical Report, no.44, Max Planck Institut für biologische Kybernetik, (1998)

[5] V.N. Vapnik, "The nature of statistical learning theory second edition," Springer, (2000)

[6] K. Sohara and M. Kotani, "Application of kernel principal components analysis to pattern recognitions," SICE2002, Aug.5-7, (2002) DOI: 10.1109/SICE.2002.1195250

[7] K. Y. Park, "Hardware realization of voice dialing system by Korean digit speech recognition," Ph.D. dissertation, Chonbuk Univ., (2001)

[8] E.B. Jung, S.M. Hong, and K.Y. Park, "Smartphone ubiquitous healthcare diagnosis system," IJCA, vol.10, no.5, pp.71-82, (2017)

[9] K.Y. Park, "Hardware construction of visual diagnosis system," KICS, Honam Section, vol.15, no.1, pp.11-17, (2006)

[10] K.Y. Park, "Wireless diagnosis system for chest disease using thoracic sound," IEEE, Honam Section, vol.13, no.1, pp.57-62, (2004)

[11] E.B. Jeoung, S.M. Hong, and K.Y. Park, "Ubiquitous healthcare diagnosis system for smartphone using vital integrated medical instrument," Proceedings of the 3rd International Mega-Conference on Green and Smart Technology, Jeju Island, Korea, December 21-23, (2016) DOI:10.14257/astl.2016.141.48 


\section{Authors}

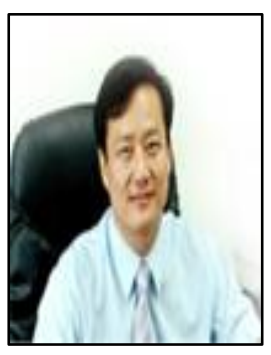

\section{Eui-Bung Jeoung}

Professor, Department of Automotive \& Mechanical Engineering, Howon University, Kunsan, Korea

<Significant area of interest: Digital Signal Processing, IOT>

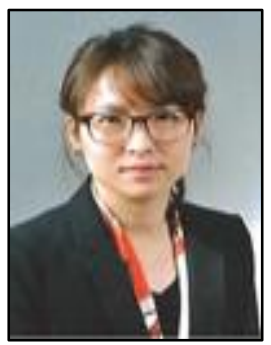

\section{Soo-mi Hong}

Assistant Professor, Department of Public Health Administration Vision College of Jeonju, Korea

<Significant area of interest: u-Healthcare system, IoT>

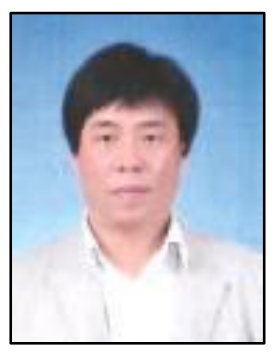

\section{Hyung-ki Choi}

Senior Researcher, Healthcare Lab., Sunmeditec co., LTD, Jeonju, Korea

$<$ Significant area of interest: Signal Processing, u-Healthcare system, IoT $>$

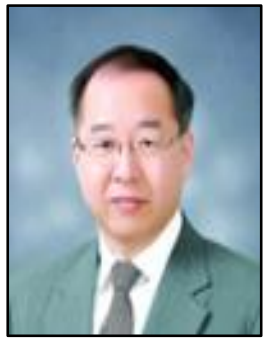

\section{Kee-young Park}

Healthcare Lab., Sunmeditec co., LTD, Jeonju, Korea

$<$ Significant area of interest: Signal Processing, u-Healthcare system, IoT $>$ 